\title{
Editorials
}

\section{Achieving earlier diagnosis of symptomatic cervical cancer}

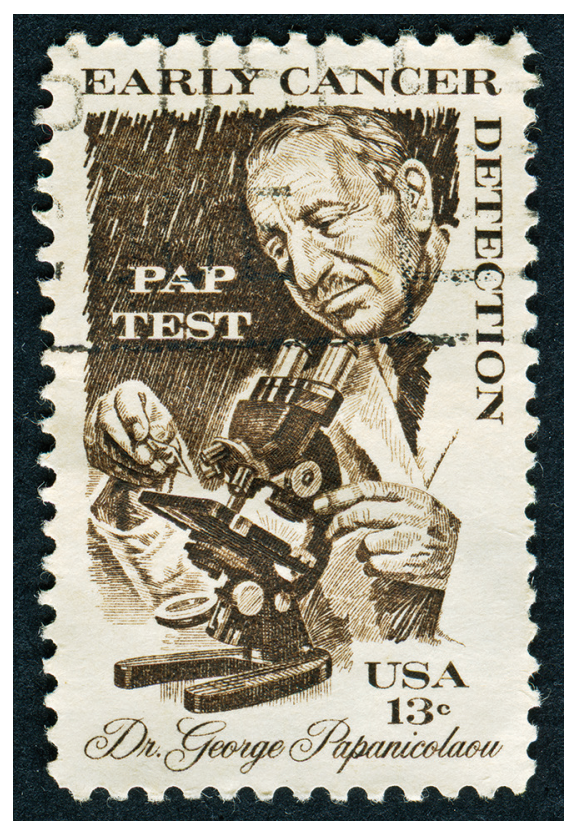

Gynaecological cancers have a combined annual incidence second only to breast cancer among women in the UK. Cervical cancer is the third most common cancer after uterine and ovarian cancer with approximately 3100 new cases and just under 1000 deaths annually in the UK. It is the most common cancer in women aged $<35$ years, and over $75 \%$ of UK cases are diagnosed in women aged $<65$ years. Cervical cancer survival is higher among women diagnosed at a younger age. The 5-year UK survival percentage is $67 \%$ overall, and almost $90 \%$ for women < 40 years of age. ${ }^{1}$ However, poorer outcomes in the UK compared with other western European countries have been well documented, and there is increasing evidence that earlier diagnosis of cervical, uterine, and ovarian cancers could contribute to a reduction in the survival gap between UK and European averages. ${ }^{2}$

\section{SYMPTOMATIC PRESENTATION}

Uterine and ovarian cancers are known to mainly present symptomatically, however, the UK's long established cervical cancer screening programme has led to a widelyheld belief among both patients and some primary care providers that the vast majority of cervical cancers are identified via screening and before symptoms develop. Cervical cancers are, indeed, identified through screening programmes, however,

the UK's long established cervical cancer screening programme has led to a widely-held belief ... that the vast majority of cervical cancers are identified via screening ... however ... many women with cervical cancer do present with symptoms...

a study by Lim and colleagues in this issue of the BJGP clearly shows that many women with cervical cancer do present with symptoms, such as vaginal bleeding, vaginal discharge, and abdominal pain. Their interview study examines the determinants of diagnostic delays among young women with symptomatic cervical cancer, and reports that a significant proportion $131 \%$ in this study) were diagnosed after a symptomatic presentation. ${ }^{3}$ Furthermore, even among their participants diagnosed via screening, more than half (56\%) also reported symptoms prior to screening, and almost half of these patients had previously reported these symptoms to a health professional lalthough in retrospect many may not have been associated with the cancer). Of the patients diagnosed after symptomatic presentation, $28 \%$ reported patient intervals (from first noticing a symptom to first presentation) ${ }^{4}$ of $>3$ months, and $60 \%$ reported provider intervals (from first presentation to diagnosis) ${ }^{4}$ of $>3$ months. Delays in presentation appeared to be primarily due to the nature of the symptom, suggesting it was due to a less serious condition or normal biological change, as well as lack of awareness of cancer symptoms. Delays in primary care were partly due to non-visualisation of the cervix, and coexisting genital infections.

\section{NATIONAL RELEVANCE}

These findings have relevance not only to the UK but also to international clinicians and policy makers, particularly in low- and middle-income countries without established population-based cervical screening programmes, where most cervical cancers are diagnosed after symptoms develop. Hence, developed and less developed countries have much to learn from each other. For instance, patients with cervical cancer in many Asian and sub-Saharan countries are known to report late for health care, experience fewer treatment benefits and have poor outcomes. ${ }^{5}$ While few studies have explored patient experiences, a recent Ugandan study interviewing healthcare professionals identified that primary care providers, often lacking adequate education, facilities, and access to pathology and specialist care, could erroneously diagnose and treat infection before excluding cervical cancer. Furthermore, a number of patient and sociocultural factors influence time to diagnosis for symptomatic cervical cancer including beliefs that cervical cancer is not curable with modern medicines and fear of cancer diagnosis. ${ }^{6}$

\section{IMPROVING TIMELY DIAGNOSIS}

What steps can be taken to improve the timely diagnosis and management of symptomatic cervical cancer in the UK?

First, public health and community awareness-raising approaches, such as the Department of Health's 'Be Clear on Cancer' campaigns, can incorporate clear and appropriate information about the most important symptoms such as vaginal bleeding, vaginal discharge, and abdominal

\section{"As gynaecological symptoms are very common and the vast majority are not due to a malignancy, the information could be targeted at specific age and higher-risk groups}




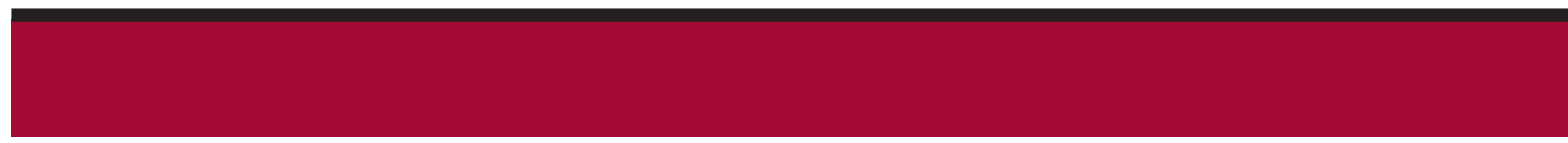

\section{"Improved management on first presentation with gynaecological symptoms may lead to reduced time to diagnosis and initiation of treatment, and possibly improved outcomes for the patient.}

pain. Such messages can also be integrated into the materials used as part of cervical screening programmes, and the materials used as part of HPV vaccination programmes and treatments. As gynaecological symptoms are very common and the vast majority are not due to a malignancy, the information could be targeted at specific age and higher-risk groups to offer personalised information for pre- and post-menopausal women, women during pregnancy, and those using hormonal forms of contraception or hormone replacement therapy. Awarenessraising campaigns can also dispel myths and stigma, and encourage discussions among women about their symptoms and what is 'normal' or appropriate for their time in life.

Lim and colleagues described a number of barriers to presentation in primary care including finding it difficult to get an appointment, worrying about not seeing a female doctor and about what the GP might find, and not wanting to waste the GP's time; ${ }^{3}$ hence these campaigns could also tackle the more general issue of how to seek help from general practice in a timely, appropriate, and acceptable way. Similar barriers have been described in many studies exploring barriers to help-seeking, and not only for cancer symptoms. Issues around embarrassment and the privacy of symptoms, particularly in minority and ethnic groups, may require a more sensitive approach for symptoms of cervical cancer. Recent similar campaigns for symptoms of colorectal and lung cancer have resulted in more consultations, investigations, and referrals, however, at present there is very little evidence that cancer awareness campaigns have any impact beyond the short to medium term. ${ }^{\text {? }}$

Second, GPs could target their female patients with similar age-and higher-risk group-appropriate information. A study published recently in the BJGP showed that most GPs would be agreeable to this sort of targeted information and promotion of earlier detection of gynaecological cancer. ${ }^{8}$ However, while GPs are generally supportive of cancer awareness-raising activities in primary care, they remain reluctant to engage in specific activities because of concerns about the potential negative impact on patients, lack of incentivisation, practice workload, and the availability of secondary care services. ${ }^{8}$

Third, GPs should examine all women presenting with symptoms of gynaecological cancer, and the NICE referral guidelines for suspected cervical cancer recommend a speculum and pelvic examination for symptomatic women. ${ }^{9}$ However there is little evidence for the value of visual inspection of the cervix in women who present to primary care with abnormal vaginal bleeding. ${ }^{10}$ Although there is also little evidence for the usefulness of cervical cytology as an aid for cervical cancer diagnosis in symptomatic women, even among women aged $<25$ years for whom cervical cytology is no longer recommended, new research may modify this recommendation. Even when examined as normal, it is important that all symptomatic patients are given appropriate safety-netting advice, such as how long to monitor symptoms, any new symptoms to prompt further help-seeking, and how to make appropriate follow-up appointments. ${ }^{11}$

Fourth, practice nurses could play a far greater role in the primary care management of women with vaginal symptoms. They now undertake the majority of cervical smears and, therefore, the routine adoption of a systematic approach to all women presenting with cervical symptoms is feasible. This could include visual inspection of the cervix, plus swabs, and cervical smear test if not previously performed within 3 years.

In conclusion, a more systematic application of current guidelines and management practice could achieve earlier diagnosis of symptomatic cervical cancer in primary care. Improved management on first presentation with gynaecological symptoms may lead to reduced time to diagnosis and initiation of treatment, and possibly improved outcomes for the patient. The medium to long-term effect of any awareness campaigns to promote symptom recognition and subsequent action remain to be fully elucidated.

\section{Fiona M Walter,}

GP and Clinician Scientist, University of Cambridge, Cambridge, UK.

\section{ADDRESS FOR CORRESPONDENCE}

Fiona Walter

The Primary Care Unit, Department of Public

Health and Primary Care, University of Cambridge,

Cambridge, CB1 8RN, UK.

\section{E-mail: fmw22amedschl.cam.ac.uk}

\section{Amos D Mwaka,}

Lecturer and Oncology Physician, Makerere University, Uganda.

\section{Richard D Neal}

Professor in Primary Care Medicine, Bangor University, Bangor, UK.

\section{Provenance}

Commissioned; not externally peer reviewed.

DOI: 10.3399/bjgp14X681649

\section{REFERENCES}

1. Cancer Research UK. Cervical cancer statistics. http://uww.cancerresearchuk.org/cancer-info/ cancerstats/types/cervix/ laccessed 12 Sep 2014).

2. Abdel-Rahman M, Stockton D, Rachet B, et al. What if cancer survival in Britain were the same as in Europe: how many deaths are avoidable? Br J Cancer 2009; 101(Suppl 2): S115-S124.

3. Lim AW, Ramirez AJ, Hamilton W. Delays in diagnosis of young females with symptomatic cervical cancer in England: an interview-based study. Br J Gen Pract 2014; DOI: 10.3399/ bjgp14X681757.

4. Weller D, Vedsted P, Rubin G, et al. The Aarhus statement: improving design and reporting of studies on early cancer diagnosis. $\mathrm{Br} \mathrm{J}$ Cancer 2012; 106(7): 1262-1267.

5. Gondos A, Chokunonga E, Brenner $\mathrm{H}$, et al Cancer survival in a southern African urban population. Int J Cancer 2004; 112(5): 860-864.

6. Mwaka AD, Wabinga HR, Mayanja-Kizza H. Mind the gaps: a qualitative study of perceptions of healthcare professionals on challenges and proposed remedies for cervical cancer helpseeking in post conflict northern Uganda. BMC Fam Pract 2013; 14: 193

7. Austoker J, Bankhead C, Forbes LJ, et al. Interventions to promote cancer awareness and early presentation: systematic review. $\mathrm{Br}$ $J$ Cancer 2009; 101(Suppl 2): S31-S39. DOI: 10.1038/sj.bjc.6605388.

8. Waller J, Evans R, Sekhon M, et al. Increasing awareness of gynaecological cancer symptoms: a GP perspective. Br J Gen Pract 2014; DOI: 10.3399/bjgp14X680161.

9. National Institute for Health and Care Excellence. Referral guidelines for suspected cancer. CG27. London: NICE, 2005.

10. Shapley M, Jordan J, Croft PR. A systematic review of postcoital bleeding and risk of cervical cancer. Br J Gen Pract 2006; 56(527): 453-460.

11. Mitchell E, Rubin G, Macleod U. Improving diagnosis of cancer: a toolkit for general practice. RCGP 2012. http://www.rcgp.org.uk/ clinical/clinical-resources/ /media/Files/CIRC/ Cancer/lmproving\%20Cancer\%20Diagnosis\%20 -\%20A\%20Toolkit\%20for\%20General\%20 Practice\%20(2).ashx (accessed 9 Sep 2014). 